\title{
Interest relativism in the best system analysis of laws
}

\author{
Max Bialek ${ }^{1,2}$
}

Received: 15 September 2015 / Accepted: 20 August 2016 / Published online: 3 September 2016

(C) The Author(s) 2016. This article is published with open access at Springerlink.com

\begin{abstract}
Lewis' Best System Analysis (BSA) of laws of nature is often criticized on the grounds that what it means to be the "best" system is too subjective for an analysis of lawhood. Recent proponents of the BSA have embraced the view's close connection to the particulars of scientific practice despite the objection. I distinguish two compatible versions of the objection: one opposed to mind or subject dependence and the other opposed to relativity. The BSA can answer both. Answering the antirelative version of Armstrong's objection requires that the BSA be no more or less relative than is required by scientific practice. A spectrum of relativity is introduced with extremes of minimally and maximally relative variants of the BSA, and extant variants of the BSA are located on it. Lastly, I sketch what work remains to be done with respect to Armstrong's objection by BSA proponents depending on where in the spectrum of relativity they hope to locate their view.
\end{abstract}

Keywords Laws of nature $\cdot$ Best systems analysis $\cdot$ Relativity $\cdot$ Scientific practice

\section{Introduction}

The Best System Analysis (BSA) of laws of nature has it, roughly, that a regularity is a law just in case it appears in the best systematization(s) of all the particular fundamental matters of fact, where the "best" means the simplest and strongest on balance (Lewis 1973, 1983, 1994, and elsewhere). A standard critique of the view,

\footnotetext{
Max Bialek

m.bialek@rug.nl

1 Faculty of Philosophy, University of Groningen, Oude Boteringestraat 52, 9712 GL Groningen, The Netherlands

2 Department of Philosophy, University of Maryland, College Park, MD, USA
} 
which I will call Armstrong's objection after its appearance as the first objection to the BSA in Armstrong (1983), is that what makes a system best is only determinable relative to a subject's conception of "best" and so, in conflict with strong intuitions that laws of nature should be objective, the laws as described by the BSA are subjective. Despite Armstrong's objection, recent proponents of the BSA (or variations of it) have tended to embrace versions of the idea that the best system is the best for us (e.g. Loewer 2007; Cohen and Callender 2009; Frisch 2011).

I argue that Armstrong's objection read as being concerned with the subject independence of the laws is deficient, since BSA laws need not depend on the existence of subjects. The specifics of Armstrong's objection as it is first presented suggest an alternative, and more threatening, reading according to which the concern is with the possibility of profligate laws determined relative to the variety of ways of filling in the details of the BSA. The two readings of Armstrong's objection are compatible and, taken together, pose a substantial challenge to the BSA with a range of possible responses. On one end of that range is the maximally relative BSA, where there are sets of laws for every possible way of filling in the details of the view. On the other end are minimally relative variants of the BSA, where limits on permissible ways of filling in the details of the view are sufficient to pick out a unique set of laws.

This paper proceeds as follows. In Sect. 2, I review the standard subject (in)dependence reading of Armstrong's objection and the case against it. In Sect. 3, I introduce the anti-relativity reading of Armstrong's objection and argue that a proper response depends on making the laws of the BSA relative exactly as much as is required by paying due respect to scientific practice. Section 4 is concerned with how the relativity of the laws of the BSA can come in degrees, and how those degrees of relativity correspond to a range of responses to the combination of the subject independence and relativity versions of Armstrong's objection. I conclude in Sect. 5 by considering where extant variants of the BSA fall in the range of more or less relative BSAs, and suggesting points that tell against adopting either extreme of relativity in future versions of the BSA.

\section{Armstrong's objection against mind/subject dependence}

Let us begin by looking at Armstrong's full statement:

The first objection that may be made to the Systematic solution [i.e. the BSA] is that an element of subjectivism remains. We have already noticed that it has to involve our standards of simplicity, which, even granted that they are shared by all rational mankind, may not be shared by other rational creatures. The same point seems to hold for standards of strength. Lewis also refers to 'our way of balancing' simplicity and strength. May there not be irresoluble conflicts about the exact point of balance? (Armstrong 1983, p. 67)

We are told at the outset that the problem is the subjectivity of the BSA laws, so let us make that part of the argument explicit:

(SBL) The laws of the BSA are subjective.

(NSL) Laws cannot be subjective. [unstated premise] 
(Conclusion) (From SBL and NSL:) The BSA cannot be a correct analysis of laws.

The argument is certainly valid. How to read "subjective" is unclear in the reconstructed argument just as it is in the passage from Armstrong. For the moment we will assume that the relevant reading of "subjective" is the one concerned with the dependence of the laws for their content and existence on the presence of minds or subjects.

The unstated premise NSL seems plausible. Proponents of the BSA might try to reject NSL from the start. One strategy for this may be to argue that it is an intuition that does not apply to laws of the BSA, in the style of the rejection in Beebee (2000) of "governing" as necessary to a conception of laws. But simply rejecting NSL weakens the standing of the view in comparison with competing accounts of laws that can accommodate the intuition. Most notably, proponents of the BSA do not reject NSL: Lewis (1994), Loewer (1996), and Cohen and Callender (2009) all (to be discussed at greater length bellow) direct their attention towards rebuffing the claim that the relation between the BSA and the standards of simplicity, strength, and their balance, makes the laws mind or subject dependent in a problematic way.

The main challenge for an employer of this objection to the BSA is to defend SBL. And, indeed, the remainder of the passage from Armstrong is dedicated to explaining just how the laws of the BSA are subjective. The matter turns on the potential for "irresoluble conflicts" between rational seekers of laws. These conflicts-call them real disagreements - are ones that persist through some ideal end of the progress of science, where nothing new could happen to decide the question and to suspend judgement would be to leave that corner of science incomplete. Armstrong suggests that a source of real disagreement may be found in the choice of measures for simplicity, strength, and their balance. The exact source of a real disagreement does not matter for our present purposes; it is enough if they occur at all. So let us grant Armstrong that there is such a real disagreement in the form of Scientist 1, who says that the BSA laws are $L_{1}$, and Scientist 2, who says that the BSA laws are $L_{2}$, where $L_{1}$ and $L_{2}$ are not equivalent. From this we are supposed to extract the claim that the laws of the BSA are subjective.

(S1L) Scientist 1 says that the BSA laws are $L_{1}$.

(S2L) Scientist 2 says that the BSA laws are $L_{2}$.

(I12) The laws $L_{1}$ and $L_{2}$ are not equivalent.

(SBL) (From S1L, S2L, and I12:) The laws of the BSA are subjective.

This argument is invalid. They each say that the BSA laws are one way or another, but that does not make them so. The BSA is, despite the inspiration it draws very directly from the epistemology of science, a realist metaphysical analysis of laws of nature. There are no variables in the BSA (like the balance between simplicity and strength) that are metaphysically free to be set at will by some subject, and so the laws of the BSA are not subjective in the sense that they depend on subjects.

I will refer to this point about the BSA as the brute metaphysics response to Armstrong's objection. Employing it is to put one's foot down and insist that there just is some metaphysical fact of the matter about what the laws are according to the BSA. It does not matter what anyone says or thinks about the laws, nor does it matter if 
anyone exists to say or think anything about the laws. Lewis adopts this position when he writes

I am talking about how nature [...] determines what's true about the laws and chances. Whether there are any believers living in the lawful and chancy world has nothing to do with it (Lewis 1994, pp. 481-482).

Cohen and Callender similarly insist in their discussion of their variant of the BSA, "relativized MRL", that

relativized MRL does not make the laws subjective in the sense that they depend for their existence on subjects. This is because the laws (relative to basic kinds $K$ ) that hold of a world $w$ would satisfy relativized MRL's criterion for lawhood...whether or not there are subjects in $w$ (or any other world) (Cohen and Callender 2009, p. 30).

Even Ramsey, whose view is summarized in Lewis (1973) as "a counterfactual about omniscience", writes that what matters to the laws are "the facts that form the system in virtue of internal relations, not people's beliefs in them" (Ramsey 1928, p. 132).

It is possible to formulate a version of the BSA that is thoroughly subjective, but it is by no means necessary. It is certainly not the case for the version of the BSA that was Armstrong's target that its laws were subjective in the sense that they depend on minds/subjects. As long as we read Armstrong's objection this way, it is less an objection than a stern reminder that we should be careful in our statements of the BSA and its variants.

\section{Armstrong's objection against relativity}

Taking a second, sympathetic, look at Armstrong and Lewis tells against interpreting Armstrong's objection as being exclusively about mind/subject (in)dependence. Armstrong never accused the BSA of being subjective, but only that, on account of (real) disagreements in scientific practice, "an element of subjectivism remains" (emphasis added). In light of this and what was said in the preceding section, it may be worth giving Armstrong the benefit of the doubt by not assuming he was objecting simply to a subject dependence in the laws.

Consider next how Lewis responds to Armstrong. Lewis' expression of the brute metaphysics sentiment is not presented as a response to Armstrong, but only as a general remark about the BSA. He does, however, write

The worst problem about the best-system analysis is that when we ask where the standards of simplicity and strength and balance come from, the answer may seem to be that they come from us. [...] Maybe some of the exchange rates between simplicity, etc., are a psychological matter, but not just anything goes. If nature is kind, the best system will be robustly best—-so far ahead of its rivals that it will come out first under any standard of simplicity and strength and balance (Lewis 1994, p. 479).

This nature is kind response to Armstrong's objection stops just short of denying that there will ever be the problematic sort of disagreement with which Armstrong is 
concerned. If nature is kind, we can deny the conjunction of S1L, S2L, and I12. But what if nature is unkind? Lewis says just down the page "in this unfortunate case there would be no very good deservers of the name of laws". This may be true if nature is wildly unkind, such that no account of laws can make sense of what is going on. The real threat to Lewis is that nature is unkind in just the right sort of way to make the BSA laws untenable but the laws as described by some other view acceptable; e.g. stochastic governing laws might allow for quite misleading/unkind runs of events in nature without undermining how deserving its laws are to be so called, and similarly for the impoverished possible worlds of Tooley (1977) and others. Such a particular threat must be warded off with more than wishful thinking.

What is most telling against the subjectivity reading of Armstrong's objection in this response from Lewis is the suggestion that the laws of the BSA may depend in principle on law seeking subjects. No amount of kindness in nature will break the subject dependence of the laws if it is there. The same issue arises with the rigidification response, considered briefly in Lewis (1986), according to which the standards of simplicity, strength, and balance employed in the BSA are fixed to "our actual standards" (Lewis 1986, p. 123). Giving Lewis the benefit of the doubt, there must be something other than subject dependence addressed by these responses.

What Armstrong and Lewis share is a concern with the presence of multiple different sets of laws, not subject dependence. The intuition underwriting Armstrong's objection is less the unstated premise (NSL) that the laws cannot be subjective (i.e. dependent on subjects), and more that the laws must be objective, which may also be interpreted as a requirement that the BSA pick out a unique set of laws. Armstrong's objection, then, is

(S1L) Scientist 1 says that the BSA laws are $L_{1}$.

(S2L) Scientist 2 says that the BSA laws are $L_{2}$.

(I12) The laws $L_{1}$ and $L_{2}$ are not equivalent.

(DBL) (From S1L, S2L, and I12:) The BSA yields multiple sets of laws.

(USL) There must be a unique set of laws.

(Conclusion) (From DBL and USL:) The BSA cannot be a correct analysis of laws.

But this is still invalid. Now, as before, we can appeal to a version of the brute metaphysics response to block any concern about extra sets of laws: The BSA will say that there is exactly one real set of laws, and so at least one of Scientist 1 and Scientist 2 is wrong.

The remainder of this section is broken up into three parts. The first is concerned with salvaging what one may from (and for) this version of Armstrong's objection by forcing the BSA to accommodate through relativity the disagreement between Scientists 1 and 2. In the second part it is shown how the BSA can be simultaneously relative and non-subjective. The third part is concerned with what would count as a response to Armstrong's objection.

\subsection{Respecting scientific practice}

It is easy to be unhappy with the version of the brute metaphysics response that was just employed. Proponents of the BSA should be unhappy because, in requiring that 
one of the two scientists be wrong, the response risks abandoning the close connection between the metaphysics of laws and the epistemology of the search for laws that is such an appealing feature of the BSA. That feature has not been downplayed in variants of the BSA proposed since Lewis: "we understand the notion of a Best System flexibly according to our needs" (Cohen and Callender 2009, p. 21); "The PDA [a variant of the BSA] goes farther in the direction of anthropo[centrism]... in that what counts as a final theory depends on the tradition of fundamental physics" (Loewer 2007, p. 325); and, in a version of the BSA concerned with chances instead of laws, "the 'best' in my use of 'Best System' means best for us" (Hoefer 2007, p. 571, emphasis in original). These authors present the close connection between their versions of the BSA and scientific practice as a virtue. But that virtue is in conflict with the brute metaphysics response as it has just been used: to dismiss some part of the practice of science.

Opponents of the BSA should also be unhappy with this version of the brute metaphysics response because it denies that the claims about laws made by the two scientists are the sort of thing that could count against the BSA. Suppose, for example, that a petulant employer of the brute metaphysics response just insists that the details of the BSA - e.g. the preferred balance between simplicity and strength, and what measures are used to determine the simplicity and strength of a system - are such that the laws are $L_{1}$. An employer of Armstrong's objection would like to be able to object: Why aren't the laws $L_{2}$ ? Nothing has been said, and, by assumption, nothing can be said, in favor of Scientist 1's settings of the details BSA over Scientist 2's. If BSA proponents are as interested as it seems in a close connection between the details of the BSA and the particulars of scientific practice, on what grounds could the position of Scientist 2 be dismissed as it has been? Some other petulant proponent of the BSA may decided to favor the details that accord with Scientist 2 over Scientist 1, or endorse some third option that is incompatible with anything in scientific practice. In any of these cases, the brute metaphysics response as just presented will ward off any criticism.

So proponents of the BSA and employers of Armstrong's objection share an interest in thwarting this version of the brute metaphysics response. Its arbitrariness and apparent disinterest in scientific practice is anathema to the methods of philosophy of science. The solution is clear: Everyone should (and should be willing to) endorse the principle that the consequences of the BSA (or any account of laws) fit, parallel, conform to, answer to, (or, to just pick a word and stick with it) respect scientific practice. Thus we add to the argument of Armstrong's objection the premise

(RSP) An account of laws must respect scientific practice.

What does RSP actually require? Saying exactly is difficult. van Fraassen explains that an account of laws

should make it plausible that laws of nature are the truths which science aims to discover. My phrasing should not be too strictly or prejudicially construed. If the account makes it plausible that the laws, as defined, are part of the theoretical description of the world provided by science in the long run, if all goes ideally well-that is enough (van Fraassen 1989, p. 55). 
For our purposes it should be emphasized that RSP requires that, whenever scientists say $X$ about laws, the BSA is in a position to agree that $X$, or at least illuminate why $X$ was said. For example, we may not have yet discovered the true BSA laws of the world, but if the BSA were run given just what we know, then it should say pretty much what we already think. Another example: The premise (USL) that there can only be one set of laws might be essential to the conception of laws found in scientific practice. In that case, USL follows directly from RSP, and the BSA should not generate multiple sets of laws.

Unlike in the case of the petulant employer of the brute metaphysics response, the BSA is now able to fail by its own lights if satisfying RSP leads to contradictory commitments. Of particular interest to the discussion of Armstrong's objection is what $\mathrm{RSP}$ requires of the BSA in the event of real disagreements in scientific practicerecall from the previous section that those are ones that persist through some ideal end of the progress of science-like in our example of Scientists 1 and 2 and their not equivalent sets of laws $L_{1}$ and $L_{2}$. Satisfying RSP will require the BSA to say that the laws of $L_{1}$ are real (not just possible/candidate) laws and the laws of $L_{2}$ are real laws; that is how the inference from S1L, S2L, and I12 to DBL is properly made. But, on pain of contradiction (since $L_{1}$ and $L_{2}$ are not equivalent), it must be qualified that each set of laws are the laws relative to something.

\subsection{Relativity and subjectivity}

The first reading of Armstrong's objection was opposed to laws that were dependent on subjects, but was set aside on account of the brute metaphysics response. But after adding RSP and requiring the the laws be relative, it is possible that the first reading of the objection becomes relevant again. If the laws of the BSA are determined explicitly relative to the peculiarities of particular scientists, then it seems that they do actually depend on subjects. Before moving on with the discussion of this second reading of Armstrong's objection, it is important to ensure that the BSA has not already succumbed to the first reading. To do that we must answer the question "To what are the BSA laws relative?".

Scientists 1 and 2 presumably don't just disagree about the laws for no reason. In Armstrong's example three points of disagreement are suggested: They might disagree on the meaning of "simple", on the meaning of "strong", and on the weighting between them that determines the overall goodness of a system. Since we have denied the possibility that there is a correct side in these disagreements (supposing that any one, or some combination, of them is the reason for the real disagreement about the laws between Scientists 1 and 2), I will say that the reasons for real disagreements are differences in interests. Scientist 1 may be interested in simple laws, while Scientist 2 is interested in strong laws. Scientist 1 may be interested in what the laws are when using one measure of simplicity, while Scientist 2 is interested in the laws when using some other measure. Since their disagreement is real, neither could ever convince the other to change their interests. But that's okay at least because each can appreciate that, were their interests to coincide, they would agree on the laws. 
We thus want the laws to be interest relative. Interest relative laws guarantee satisfying RSP as long as any differences of interest may be reflected in how the details of the BSA are filled in. But the laws of the BSA cannot be explicitly interest relative since interests are had by subjects and that would make the laws of the BSA subjective in conflict with the first reading of Armstrong's objection. Suppose that the BSA run with details $D_{1}$ yields the laws $L_{1}$. And, if run with details $D_{2}$, the BSA yields the laws $L_{2}$. Nothing about these relations realized by the BSA, between the details $D_{i}$ and corresponding laws $L_{i}$, result in laws that are subject dependent. The $L_{i}$ are out there just as the non-relative BSA laws were when we first considered how the brute metaphysics response blocks the first reading of Armstrong's objection. What has changed is this: There are not just the laws. There are the laws of Scientist 1, and the laws of Scientist 2, and so on if need be.

To say that the laws $L_{1}$ are the laws of Scientist 1 certainly makes it sound like the laws depend on Scientist 1, but the dependence need not be present. The laws $L_{1}$ are those relative to the details $D_{1}$, whether there are any subjects or not. When Scientist 1 comes along with interests $I_{1}$, those interests point to the already present laws $L_{1}$-determined relative to details $D_{1}$ that correspond to the interests $I_{1}$-as the laws of interest to Scientist 1. This is a generalized version of the picture I assume one must have in order to reconcile the relative laws of Cohen and Callender (2009) (to be discussed further in Sect. 5) with their claims to subject independence.

We can say that the laws are interest relative insofar as there are different ways of filling in the details of the BSA (to which the laws are actually relative) corresponding to the different interests of scientists. In this way the laws of the BSA can be interest relative to satisfy RSP without succumbing to subjectivity.

\subsection{Responding to Armstrong}

Now we may return to addressing the second reading of Armstrong's objection. Can the laws of the BSA be relative and guarantee a unique set of laws (as required by USL)? Yes, in two possible ways. The first way is what was suggested by Lewis in the "nature is kind" response. It may be, if nature is kind, that all the sets of laws (each relative to the different ways of fixing the free variables of the BSA) turn out to be equivalent, and thus there is effectively just one set of laws yielded by the BSA. But we have already set aside this possibility as too much wishful thinking. The second way is if, in fact, there are no real disagreements or differences of interest in scientific practice. However plausible this may or may not be, it amounts to a denial of the conjunction of S1L, S2L, and I12, and so to simply assert it is question begging as a response to Armstrong's objection.

If there are real disagreements in scientific practice, then the BSA will fail to satisfy USL when it tries to satisfy RSP by admitting relative laws that accommodate those disagreements. But, at the same time, the justification for USL will be undermined by RSP since it is scientific practice itself that is requiring that there be multiple sets of laws. In light of asserting that there is real disagreement among scientists (premises S1L, S2L, and I12) and that the BSA must respect scientific practice (RSP), one cannot insist on there being a unique set of laws (USL). It is not that there can only be one 
set of laws, but rather that there should be only as many sets of laws as is required by respecting scientific practice. Since multiple sets of laws are yielded by allowing the laws of the BSA to be relative to corresponding details, we may say that the BSA is more or less relative when it admits more or fewer sets of laws (we'll see, though, that this connection comes apart a bit in Sect. 4). One would need to introduce (and defend) principles that supersede RSP in order to request or endorse any more or less relativity (or more or fewer sets of laws) than what is required to satisfy RSP.

Before moving on, let us pause to take stock of what has been said and see where it may take us. There are two (related) intuitions underlying Armstrong's objection, each a version of the intuition that laws should be objective. The first is that the laws cannot be subject dependent, and is satisfied by the BSA with little issue. The second is that there should be just one set of laws. Making the second intuition problematic for the BSA requires endorsing the claim that an analysis of laws must respect scientific practice and guaranteeing that there are real disagreements in scientific practice that would force (on account of respecting scientific practice) the BSA to allow for the existence of more than one set of laws. If the two intuitions are to be defended, it is presumably by examination of scientific practice. Therefore, insofar as scientific practice requires more than one set of laws, the second intuition must be weakened. It is not that there must be just one set of laws. Rather, there should be only as much relativity in the laws as is required by respecting scientific practice. Responding to Armstrong's objection is thus a matter of finding just the right amount of relativity in the laws of the BSA. Determining how relative the laws of the BSA should be is a project beyond the scope of this paper, but in the remaining two sections I address how the BSA can be more or less relative, and what that means for present and future variants of the view.

\section{Degrees of relativity}

The BSA may be illuminated by a story in which a scientist dies and appears before God, who asks if there is anything the scientist would like. The scientist replies "I would like to know how the world works". God accepts the request and begins "At time and position $\left\langle t_{1}, p_{1}\right\rangle$ such-and-such properties obtained, and at $\left\langle t_{1}, p_{2}\right\rangle$ such-andsuch other properties obtained, and-_" at which point the scientist interrupts and says "Sorry, if that is how things actually work that is all well and good, but is there a pithy version?". God's reply to that question is to provide the scientist with the (BSA-style) laws of nature. Beebee (2000) introduces a version of this story in order to highlight the fact that the laws of the BSA are non-governing. It also provides a nice context for thinking about how the laws of the BSA are relative. All the same questions are raised when the scientist asks for the "pithy version" as when it is said that the laws are drawn from the "best system". And if two scientists appear before God and receive different laws, that is akin to our earlier thinking about real disagreements between scientists at some ideal end of science. Thinking of Armstrong's objection as being concerned with relativity, the question to ask is "How different are the respective laws?".

Differences in the laws are realized by different ways of filling in the details of the BSA. Earlier this was likened to setting a metaphysically free variable like the balance 
between simplicity and strength. Let us make that more explicit with a toy model of the BSA that is as naive and straightforward as possible: A system $S$ is judged best (or not) according to its goodness $\operatorname{Good}(S)$ which is the sum of its simplicity $\operatorname{Simp}(S)$ and strength $\operatorname{Str}(S)$ weighted by a balance factor $b$ with a real value from 0 to 1 such that

$$
\operatorname{Good}(S)=b \times \operatorname{Simp}(S)+(1-b) \times \operatorname{Str}(S)
$$

Suppose further that the measures Simp and Str are fixed so that there is only the one free variable $b$. The BSA relativized to $b$ says that there are laws $L_{b=x}$ for each way of substituting a value $x$ in for $b$.

We can ask again: How different are the respective laws? Without saying anything about what the laws look like in detail, it will be hard to talk of the difference between any two sets of laws $L_{b=x}$ and $L_{b=y}$ (with $x \neq y$ ) beyond simple equality or inequality. We can make some progress, though, in talking about the degree of relativity of the set of all law sets $\mathcal{L}=\left\{L_{b=x} \mid\right.$ for all $x \in \mathbb{R}$ such that $\left.0 \leq x \leq 1\right\}$.

When Lewis introduces the "nature is kind" response it is meant to minimize relativity by having $\mathcal{L}$ have a single member as a result of the fact that $L_{b=x}=L_{b=y}$ for all $x, y$. As soon as there is not one all encompassing equivalence class of law sets there is an abrupt jump from there being effectively no relativity in the laws to there being some (e.g. if there is some critical boundary value of balance $v$ such that $L_{b=x}=L_{b=y}$ iff either $v \leq x, y$ or $x, y<v$ ).

In the limit where $L_{b=x} \neq L_{b=y}$ for all $x, y$ such that $x \neq y$, there are uncountably many distinct law sets. Relativity goes down from that point in one of two ways. The first is as we've built up to this point in reverse: If there are any equivalent sets of laws, then it seems there is less relativity. For example, if there is some value of balance $v$ such that $L_{b=x}=L_{b=y}$ iff $v \leq x, y$. Then, unless $v=0$, there will still be uncountably many nonequivalent law sets. But the claim that the laws will come out the same as long as a weight of at least $v$ is given to simplicity seems to constitute a substantial reduction in relativity compared to every value of $b$ yielding different laws.

The second way for relativity to go down is if there are additional constraints on the value of $b$. It is assumed that $b$ has some real value ranging from 0 to 1 . What if it could be shown that the extreme values are not allowed, that $b$ must have a real value between 0 and 1 ? Again, there are still uncountably many nonequivalent law sets-only two distinct law sets have been excluded. But, again, the claim that the goodness of a system cannot be determined entirely by its simplicity or entirely by its strength seems substantial as a constraint on relativity.

The implausibility of the "nature is kind" scenario is tempered by the possibility of identifying equivalencies and limiting the set of permissible values $b$. There will be just one set of laws as long as all of the balance relative laws are equivalent for any permissible value of $b$. That is minimal relativity (at least in the limited context of relativity to $b$ ). It is, presumably, what one has in mind when they insist that the laws of the BSA be objective in the strong sense of being opposed to the possibility of any disagreement.

Is there a point at which the relativity of the BSA cannot be increased? It cannot just be that maximum relativity is achieved where there is an uncountable infinity of 
nonequivalent law sets. We can keep increasing the relativity of the laws by letting more and more of the BSA be variable. One step in that direction would be to allow for many ways of measuring each of simplicity, strength, and fit. But the potential for variability in the details of the BSA runs much deeper than just how we measure and balance the good-making features of a system. Lewis' BSA and the variants of the view introduced since all share four parts: First, there are the matters of fact to be systematized. Second, the various candidate systems. Third, all the languages that might be used to express the facts and candidate systems. Fourth and finally, the best system competition itself, which serves as a function from the sets of facts, systems, and languages, to a system-language pair (or set of such pairs) that is the source of the laws. When we consider laws that are relative to the exact balance of simplicity and strength, this is a worry about the rules of the best system competition. But there could also be relativity to how the set of facts to be systematized is populated. And relativity to the set of systems that will be considered as candidates in the competition. And relativity to the set of languages in which the systems and facts may be expressed.

Maximal relativity is achieved when all four parts of the BSA - the facts, systems, languages, and competition-are wholly unconstrained beyond the loose structure that brings those four parts together. Between the maximally relative BSA and the minimally relative BSAs there is a wide range of BSA variants that feature a middling amount of relativity. And hopefully among those, or among the extreme variants if that is how things go, is a variant that captures exactly the relativity, no more and no less, that is required by respecting scientific practice. If there is, then Armstrong's objection as it has been reconstructed in this paper can be answered.

\section{Conclusion: past and future BSAs}

The purpose of this paper has been to provide an analysis of Armstrong's objection to the BSA and show that it may be answered by a variety of BSA variants. Armstrong's objection had two possible readings: The first, which objects to laws being mind or subject dependent, was found wanting because the laws of the BSA need not be mind/subject dependent. The second reading, which objects to there being more than one actual set of laws, is only able to require of the BSA that its relativity match exactly what is required by scientific practice. There is a spectrum of variously relative BSA variants, from a single maximally relative variant that lets sets of laws be identified relative to any way of filling in the details of the BSA, all the way down to a host of minimally relative BSAs that each identify only a single set of laws.

This range is largely covered by extant variants of the BSA. Lewis, as indicated by his introduction of the "nature is kind" response, pursued a minimally relative BSA. Loewer seems to be seeking a minimally relative BSA; Loewer (2012) joins Albert (2000) in identifying a single set of laws, but those are explicitly the fundamental laws and so there may still be space in their view to admit other sets of (non-fundamental) laws. Cohen and Callender (2009) have come the closest to maximal relativity. The centerpiece of their version of the BSA is the relativizing of laws to what kinds are treated as basic in the language(s) that are used to express the facts and competing 
systems. Competition relativity is also a part of their view, though less discussed than the kind relativity; it is explained that

Ecologists are not looking over their shoulders at the simplicity, strength and balance metrics of physics. They are using their own metrics tailored to their own field (Cohen and Callender 2009, p. 24).

And similar reasoning probably extends to what counts as a candidate system; e.g., ecologists probably are not counting field theories among their candidate systems. All that is missing from their view is a relativity to what facts are to be systematized, and nothing about the view tells against its introduction.

If respecting scientific practice requires that there be laws for every scientific field, then it seems the BSA may be pushed quickly towards maximal relativity. But other considerations, still introduced in the interest of respecting scientific practice, might serve to limit relativity. Desiderata for accounts of laws may impose limits; e.g. Lewis (1983) argues that certain gerrymandered predicates must be excluded from the considered languages in order to guarantee that the laws distinguishes between lawful and accidental regularities. And think again of the story of a scientist appearing before God and receiving the laws of nature. It does a lot to illuminate the nature of BSA laws, but it gets an important feature of scientific practice wrong: Science is done in the world. The results of experiments must be written down in the same space that is (broadly speaking) the subject of the experiments. The systematic calculations of practicing scientists must be done in a way that comports with the very collection facts that are to be systematized. Some costs—in time, space, and energy —of doing science in the world must be inescapable. If all scientists aspire to be successful, then they will not pursue lines of inquiry that are guaranteed to fail on account of their costs; e.g., competition functions that are incalculable, or system-language pairings that would require more space than is available in the world to express.

If one's goal is to find a minimally relative variant of the BSA, then a lot of work must be done to either (1) reject the gains in respecting scientific practice achieved by a view like that of Cohen and Callender, or (2) identify limits on relativity that would overcome the increases in relativity required to achieve admitted gains in respecting scientific practice. If one's goal is to defend the maximally relative BSA, then something must be said about why there are no means of limiting relativity to be found in scientific practice. If one's goal is to use Armstrong's objection against the BSA, then the tasks that now face the minimal and maximal relativists must be done in tandem until there is no room for any coherent amount of relativity in the BSA. Answering Armstrong's objection requires neither a deferent minimally relative variant of the BSA, or the defiant maximally relative variant of the BSA. Something in between may be just right, with relativity, and limits to it, properly motivated by appeals to scientific practice.

Acknowledgements I would like to thank Leah Henderson, Barry Loewer, Aidan Lyon, and Jan-Willem Romeijn for their comments and support leading up to and during the development of this paper. Thanks also go to the organizers and audience at the 8th Munich-Sydney-Tilburg Conference in Philosophy of Science, an audience at the 2015 meeting of the European Philosophy of Science Association, and three anonymous referees. 
Open Access This article is distributed under the terms of the Creative Commons Attribution 4.0 International License (http://creativecommons.org/licenses/by/4.0/), which permits unrestricted use, distribution, and reproduction in any medium, provided you give appropriate credit to the original author(s) and the source, provide a link to the Creative Commons license, and indicate if changes were made.

\section{References}

Albert, D. Z. (2000). Time and chance. Cambrdige, MA: Harvard University Press.

Armstrong, D. M. (1983). What is a law of nature? Cambridge: Cambridge University Press.

Beebee, H. (2000). The non-governing conception of laws of nature. Philosophical and Phenomenological Research, 61, 571-594.

Cohen, J., \& Callender, C. (2009). A better best system account of lawhood. Philosophical Studies, 145(1), $1-34$.

Frisch, M. (2011). From arbuthnot to boltzmann: The past hypothesis, the best system, and the special sciences. Philosophy of Science, 78(5), 1001-1011.

Hoefer, C. (2007). The third way on objective probability: A sceptic's guide to objective chance. Mind, 116(463), 549-596.

Lewis, D. (1973). Counterfactuals. Malden, MA: Blackwell Publishers.

Lewis, D. (1983). New work for a theory of universals. Australasian Journal of Philosophy, 61(4), 343-377.

Lewis, D. (1986). Philosophical Papers (Vol. II). Oxford: Oxford University Press.

Lewis, D. (1994). Humean supervenience debugged. Mind, 103(412), 473-490.

Loewer, B. (1996). Humean supervenience. Philosophical Topics, 24(1), 101-127.

Loewer, B. (2007). Laws and natural properties. Philosophical Topics, 35(1/2), 313-328.

Loewer, B. (2012). Two accounts of laws and time. Philosophical Studies, 160(1), 115-137.

Ramsey, F. P. (1928). Universals of law and of fact. In D. H. Mellor (Ed.), Foundations: Essays in philosophy, logic, mathematics and economics (pp. 128-132). London: Routledge \& Kegan Paul.

Tooley, M. (1977). The nature of laws. Canadian Journal of Philosophy, 7(4), 667-698.

van Fraassen, B. C. (1989). Laws and symmetry. Oxford: Oxford University Press. 